\title{
Restoration of hematopoiesis in a case of myelodysplastic syndrome associated with systemic lupus erythematosus treated with rituximab
}

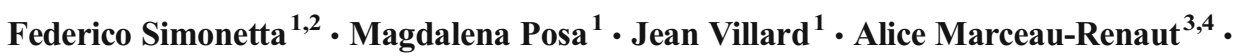 \\ Claude Preudhomme ${ }^{3,4}$ - Kaveh Samii ${ }^{2}$ Carlo Chizzolini ${ }^{1}$
}

Received: 5 January 2015 / Accepted: 20 March 2015 /Published online: 7 April 2015

(C) Springer-Verlag Berlin Heidelberg 2015

Dear Editor,

Experimental and clinical evidence indicates that autoimmune mechanisms contribute to the pathogenesis of cytopenias in some cases of myelodysplastic syndrome (MDS). Dysregulation in inflammatory cytokine production, skewing of the T-cell repertoire, and specific T-cell responses directed to antigens overexpressed by dysplastic clones have been reported [1]. Furthermore, MDS are frequently associated with systemic autoimmune disorders [2, 3], and immunosuppressive regimens have been successfully employed to restore hematopoiesis in selected MDS patients [4]. A 61-year-old Caucasian woman known for a long standing SLE was admitted for shortness of breath and dizziness worsening over 2 weeks. Before admission, SLE was stable for many years in the absence of any ongoing immunosuppressive treatment although medication history included previous treatments with chlorambucil and cyclophosphamide. Clinical examination revealed pallor without jaundice, lymphadenopathy, or hepatosplenomegaly. Laboratory investigations showed severe non-regenerative macrocytic anemia (hemoglobin $(\mathrm{Hb})$ $40 \mathrm{~g} / \mathrm{l}, \mathrm{MCV} 100.9 \mathrm{fl}$, reticulocytes $\left.4.610^{9} / \mathrm{l}\right)$ with normal leukocytes (WBC $4.310^{9} / 1$ ), and platelets counts (Plt

Federico Simonetta

federico.simonetta@hcuge.ch

1 Immunology and Allergy, Department of Medical Specialties, School of Medicine, Geneva University Hospitals, Rue Gabrielle-PerretGentil 4, 1211 Geneva 14, Switzerland

2 Hematology, Department of Medical Specialties, School of Medicine, Geneva University Hospitals, Geneva, Switzerland

3 U837, INSERM, Lille, France

4 Hematology Laboratory, Biology and Pathology Center, Lille University Hospital, Lille, France $\left.29410^{9} / 1\right)$. Lactate dehydrogenase was $380 \mathrm{U} / 1$, haptoglobin $294 \mathrm{mg} / \mathrm{l}$, and unconjugated bilirubinemia $7 \mathrm{mmol} / \mathrm{l}$. Serological testing failed to detect any acute or chronic viral infection. Direct Coombs's test was strongly positive for immunoglobulin $\mathrm{G}$ and complement $\mathrm{C} 3 \mathrm{~d}$. Bone marrow examination showed severe dysplasia affecting all three lineages without blasts excess. Interestingly, we observed a severe erythroid hypoplasia associated with specific maturation block in erythroid-lineage cells which were constituted by proerythroblasts and basophilic erythroblasts in the virtual absence of polychromatophilic and orthochromatophilic erythroblasts (Fig. 1a, left panel). Cytogenetic analysis showed normal female karyotype. Molecular analysis revealed a missense mutation in the exon 6 of the $U 2 A F 1$ gene (c. $470 \mathrm{~A}>\mathrm{C}$, p.Q157P). A diagnosis of MDS of the refractory cytopenia with multilineage dysplasia (RCMD) subtype was established, and massive transfusional support was initiated with only slight increase in $\mathrm{Hb}$ levels (Fig. 1b). No alloimmunization was detected. We hypothesized a pathogenic role of warm autoantibodies in red blood cell transfusion refractoriness, and corticosteroid treatment was initiated with no improvement. According to previous reports [5], we initiated rituximab (RTX) $375 \mathrm{mg} / \mathrm{m}^{2} /$ week for 4 weeks. A prompt increase in Hb levels was observed, and by the end of RTX treatment, transfusion independence was obtained (Fig. 1b). Bone marrow examination performed 3 months after RTX treatment still showed persistent signs of three lineages dysplasia but revealed restoration of erythroid maturation with reappearance of significant numbers of polychromatophilic and orthochromatophilic erythroblasts (Fig. 1a, right panel). During follow-up, we observed stable $\mathrm{Hb}$ levels, a normalized $\mathrm{MCV}$, and sustained reticulocyte counts indicating persistence of an effective erythropoiesis. Unfortunately, 3 years after initial MDS diagnosis, the patient presented with pancytopenia due to acute myeloid leukemia (AML) transformation. 
Fig. 1 Restoration of erythropoiesis after rituximab treatment. a Bone marrow examination performed before (left panel) and after (right panel) rituximab treatment. b Evolution of hemoglobin $(\mathrm{Hb})$ levels (continuous black line) before and after rituximab cures (dashed lines). Grey-filled circles indicate red blood cell concentrate (RBC) transfusions administered
A
Before Rituximab

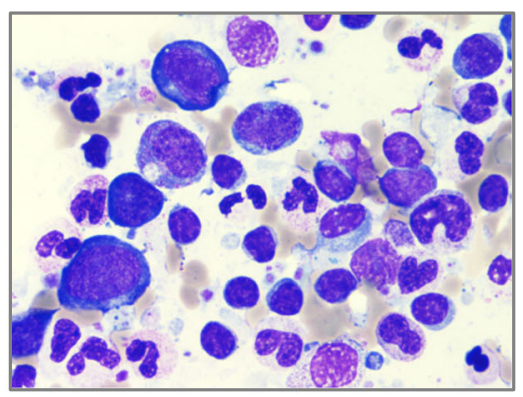

After Rituximab

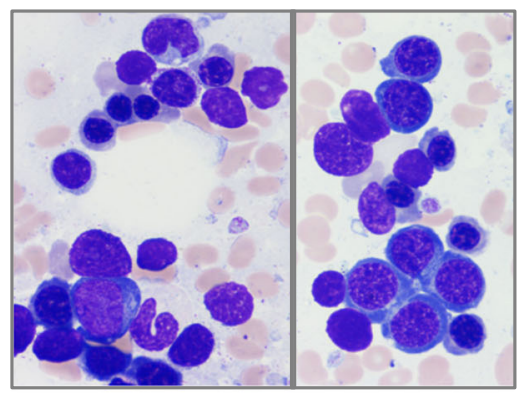

B

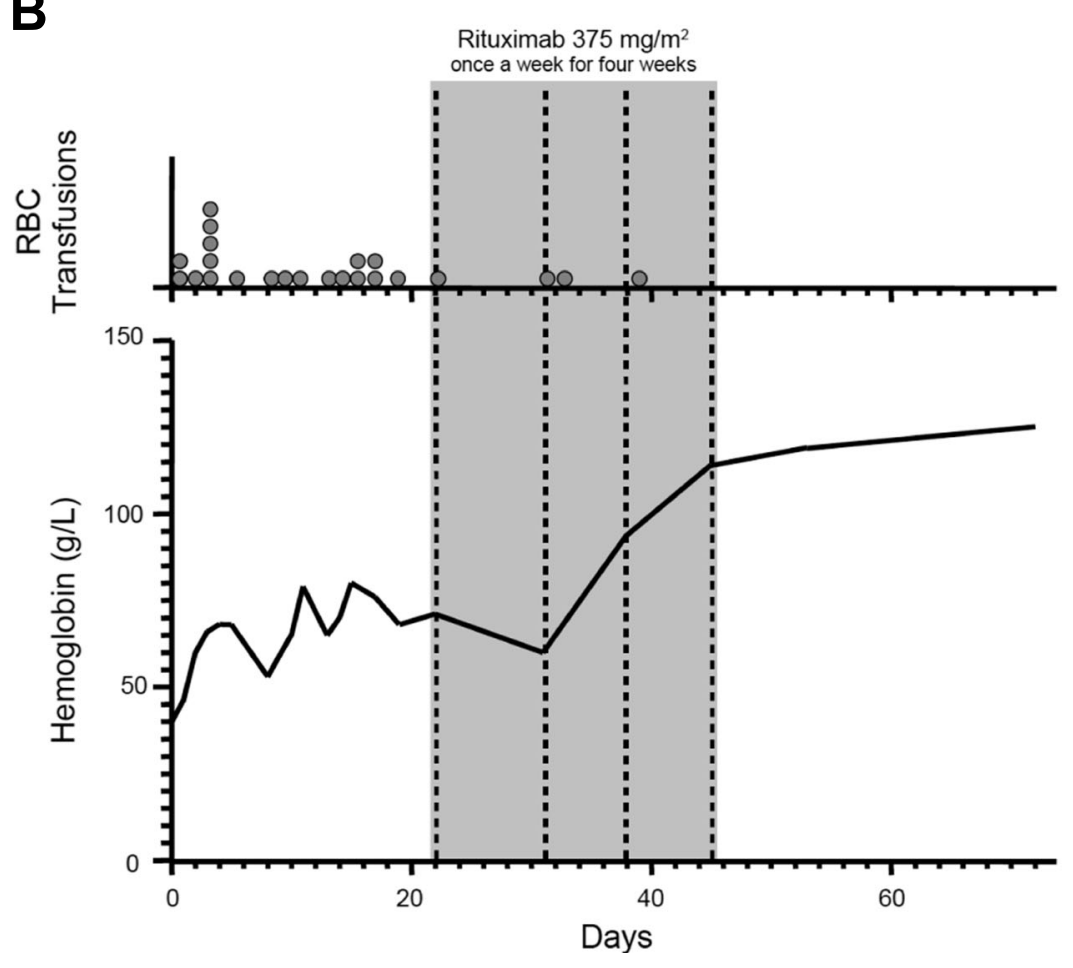

Various immunosuppressive strategies, including cyclosporin-A [6], antithymoglobulin [7] or alemtuzumab [8], proved effective in obtaining hematologic responses and transfusion independence in some patients with MDS. To the best of our knowledge, this is the first report of hematologic response of MDS to RTX, although, similarly to what previously reported for other immunosuppressive strategies [9], such treatment failed to prevent AML transformation in our patient. Our case points to B-cell depletion as a potential therapeutic strategy to induce hematologic response in selected cases of MDS.

Conflicts of interest The authors declare that they have no conflict of interest.

\section{References}

1. Barrett AJ, Sloand E (2009) Autoimmune mechanisms in the pathophysiology of myelodysplastic syndromes and their clinical relevance. Haematologica 94(4):449-51. doi:10.3324/haematol.2009. 006080

2. Giannouli S, Kanellopoulou T, Voulgarelis M (2012) Myelodysplasia and autoimmunity. Curr Opin Rheumatol 24(1): 97-102. doi:10.1097/BOR.0b013e32834db4ee

3. Lu M, Bernatsky S, Ramsey-Goldman R, Petri M, Manzi S, Urowitz MB, Gladman D, Fortin PR, Ginzler EM, Yelin E, Bae SC, Wallace DJ, Jacobsen S, Dooley MA, Peschken CA, Alarcón GS, Nived O, Gottesman L, Criswell LA, Sturfelt G, Dreyer L, Lee JL, Clarke AE (2013) Non-lymphoma hematological malignancies in systemic lupus erythematosus. Oncology 85(4):235-40. doi:10.1159/ 000350165

4. Parikh AR, Olnes MJ, Barrett AJ (2012) Immunomodulatory treatment of myelodysplastic syndromes: antithymocyte globulin, 
cyclosporine, and alemtuzumab. Semin Hematol 49(4):304-11. doi: 10.1053/j.seminhematol.2012.07.004

5. Bussone G, Ribeiro E, Dechartres A, Viallard JF, Bonnotte B, Fain O, Godeau B, Michel M (2009) Efficacy and safety of rituximab in adults' warm antibody autoimmune haemolytic anemia: retrospective analysis of 27 cases. Am J Hematol 84(3):153-7. doi:10.1002/ajh.21341

6. Jonasova A, Neuwirtova R, Cermak J, Vozobulová V, Mociková K, Sisková M, Hochová I (1998) Cyclosporin A therapy in hypoplastic MDS patients and certain refractory anaemias without hypoplastic bone marrow. Br J Haematol 100(2):304-9

7. Stadler M, Germing U, Kliche KO, Josten KM, Kuse R, Hofmann WK, Schrezenmeier H, Novotny J, Anders O, Eimermacher H, Verbeek W, Kreipe HH, Heimpel H, Aul C, Ganser A (2004) A prospective, randomised, phase II study of horse antithymocyte globulin vs rabbit antithymocyte globulin as immune-modulating therapy in patients with low-risk myelodysplastic syndromes. Leukemia 18(3):460-5

8. Sloand EM, Olnes MJ, Shenoy A, Weinstein B, Boss C, Loeliger K, Wu CO, More K, Barrett AJ, Scheinberg P, Young NS (2010) Alemtuzumab treatment of intermediate-1 myelodysplasia patients is associated with sustained improvement in blood counts and cytogenetic remissions. J Clin Oncol 28(35):5166-73. doi:10.1200/JCO. 2010.29.7010

9. Passweg JR, Giagounidis AA, Simcock M, Aul C, Dobbelstein C, Stadler M, Ossenkoppele G, Hofmann WK, Schilling K, Tichelli A, Ganser A (2011) Immunosuppressive therapy for patients with myelodysplastic syndrome: a prospective randomized multicenter phase III trial comparing antithymocyte globulin plus cyclosporine with best supportive care-SAKK 33/99. J Clin Oncol 29(3):303-9. doi:10.1200/JCO.2010.31.2686 